\title{
Design and Analysis of Tail Gas Treatment Device for High Power Diesel Engine
}

\author{
Chunyu Yu ${ }^{1,}$, , Jiaping Song ${ }^{1}$, Xiaobing $\mathrm{Zhu}^{2}$, Yong Song ${ }^{1}$ \\ ${ }^{1}$ School of Mechatronic Engineering, Southwest Petroleum University, Chengdu, China \\ ${ }^{2}$ Chengdu Jiesen Transmission and Distribution Equipment Co., Ltd., Chengdu, China
}

\section{Email address:}

ycyswpu@swpu.edu.cn (Chunyu Yu)

${ }^{*}$ Corresponding author

\section{To cite this article:}

Chunyu Yu, Jiaping Song, Xiaobing Zhu, Yong Song. Design and Analysis of Tail Gas Treatment Device for High Power Diesel Engine. International Journal of Science, Technology and Society. Vol. 5, No. 1, 2017, pp. 1-8. doi: 10.11648/j.ijsts.20170501.11

Received: February 24, 2017; Accepted: March 29, 2017; Published: April 15, 2017

\begin{abstract}
Diesel engine has the advantages of low fuel consumption, low carbon dioxide emission and high reliability, so it has been used in many fields. Particular matter (PM) and nitrogen oxide $\left(\mathrm{NO}_{\mathrm{X}}\right)$ are seriously overweight, which not only endanger people's health, but also cause environmental pollution. For the high power diesel engine, a kind of tail gas treatment device with cyclone separation and electrostatic trapping function is designed.
\end{abstract}

Keywords: Cyclone, Diesel Engine, Exhaust Gas Treatment, Numerical Simulation

\section{Introduction}

Diesel engine has the advantages of low fuel consumption, low carbon dioxide emission and high reliability, so it has been used in many fields [1]. Particular matter (PM) and nitrogen oxide $\left(\mathrm{NO}_{\mathrm{X}}\right)$ are seriously overweight, which not only endanger people's health, but also cause environmental pollution. At present, there are many ways to deal with the exhaust gas of low power diesel engines [2], mainly including chemical method and mechanical method, and the exhaust of disposal route of high power diesel engine is needed to further study.

In this paper, an electrostatic cyclone is designed for high power diesel engine. Cyclone separator has a good separation effect on large particles in the exhaust gas, at the same time, the electric has a good separation effect on the smaller diameter particles. The combination of the two can greatly improve the efficiency of separation and acquisition.

\section{Structure and Principle}

The electrostatic cyclone separation device is mainly composed of a support frame, a cyclone separator, four vibrating devices and Corona electrode. Cyclone separator adopts spiral inlet, which includes corona pole, insulator and so on. The exhaust gas containing PM particles and $\mathrm{NO}_{\mathrm{X}}$ enters into the separator from the intake pipe, and downward spiral motion due to the restriction of the vessel wall [3]. A non-uniform electric field is established between the discharge electrode and the wall of the device. When the voltage rise to a certain critical value, the air is breakdown. It can make the dust charged after air ionization, charged dust under the effect of electric field force movement in the direction of the wall [4]. At the same time, the centrifugal force generated by the rotating motion also throws the dust particles into the wall of the machine. The captured particles under gravity along the wall down to the bucket. After purification, the gas in the cone section of bottom-up research to the same spiral movement, and then through the top of the exhaust pipes.

\section{System}

\subsection{Cyclone Separator}

The cyclone separator has three technical performance indexes, such as handling air volume, pressure loss and dust removal efficiency. This cyclone separator is designed to meet the Z12V190 diesel engine. The oxygen content of safe drilling is not more than $14 \%$, The oxygen content of safe drilling is not more than $14 \%$, the maximum discharge of single $88.1 \mathrm{~m} 3 / \mathrm{min}$, the flow rate is $30 \mathrm{~m} / \mathrm{s}$. 


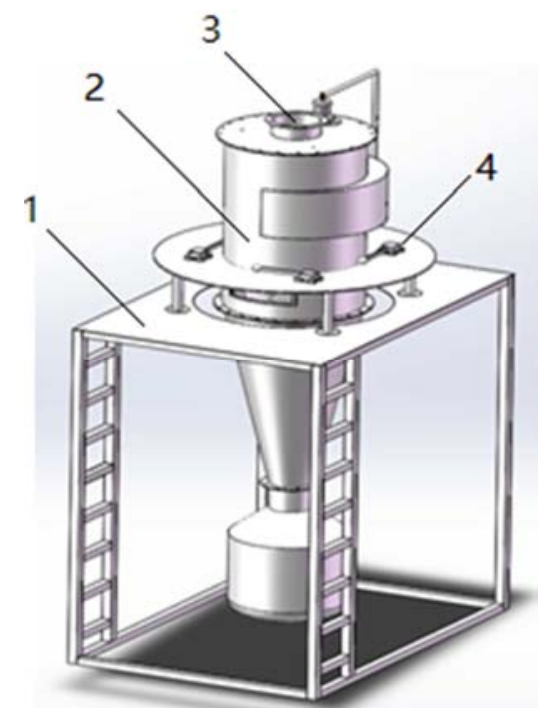

1-support frame; 2-cyclone separator; 3-corona electrode; 4-vibrating device

Figure 1. Schematic diagram of electrostatic cyclone separator.

The inlet form selects the tangential type, and the inlet pipe selects the rectangular inlet pipe [5]. This style can increase separator inlet radius, improve rotational speed, reduce the cutting size. The basic mechanism of cyclone separator is shown in Figure 2. The design parameters are shown in Table $1[6-10]$.

Table 1. The design parameters of cyclone separator.

\begin{tabular}{ll}
\hline Parameters/Symbol & Size (mm) \\
\hline Height of inlet $/ \mathrm{h}$ & 350 \\
Width of inlet $/ \mathrm{b}$ & 140 \\
Height of the cylinder $/ \mathrm{H}$ & 1300 \\
Diameter ofthe cylinder $/ \mathrm{D}_{0}$ & 700 \\
Diameter of the ash bucket $/ \mathrm{D}_{\mathrm{d}}$ & 210 \\
Height of the cone $/ \mathrm{L}$ & 1400 \\
Diameter of exhaust pipe $/ \mathrm{d}_{\mathrm{e}}$ & 245 \\
The exhaust pipe insertion depth $/ \mathrm{h}_{\mathrm{e}}$ & 680 \\
\hline
\end{tabular}
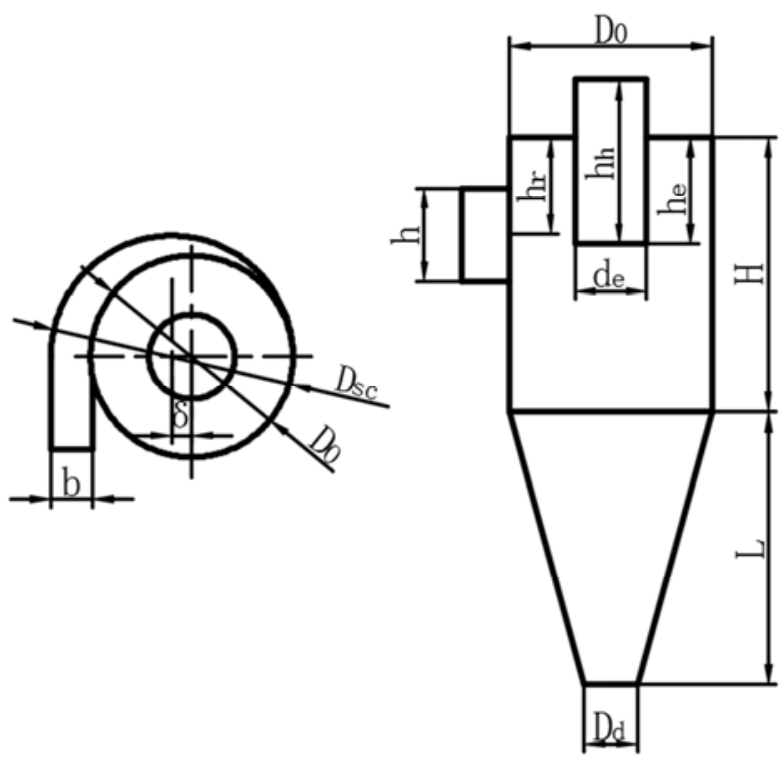

Figure 2. The basic mechanism of cyclone separator.

\subsection{Corona Electrode}

A corona electrode structure is added on the conventional separator to generate a high-voltage electric field in the separator to form an electrostatic cyclone separator. Electrostatic cyclone separator has strong ability to capture dust particles with smaller particle size [11].

Corona electrode is arranged in the outer vortex mode. The discharge line adopts RS tube shaped barbed wire. As shown in Figure 3, the corona wire is composed of a connecting piece, a hanging ring, rods, thorns and a supporting ring. In order to ensure good electrical conductivity, all structures except the awn are made of copper.

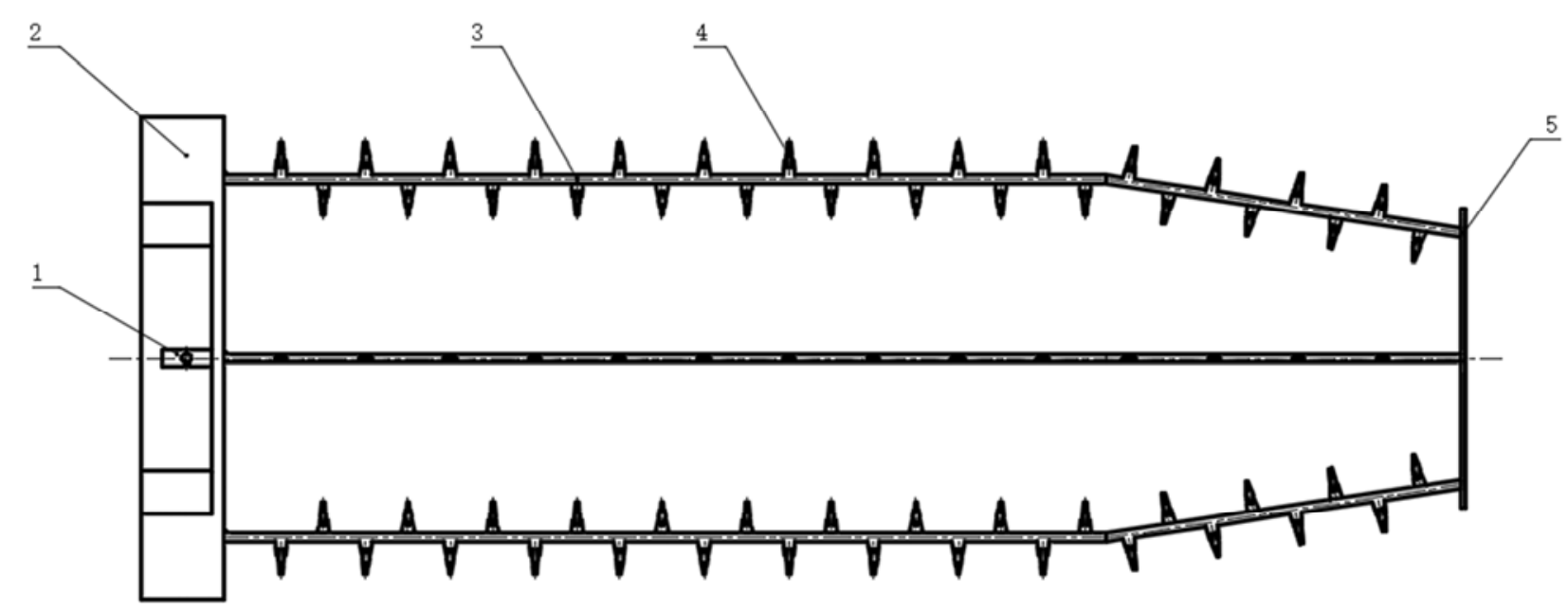

1-connecting piece; 2-hanging ring; 3- rod; 4-thorn; 5-supporting ring

Figure 3. Schematic diagram of corona electrode. 


\subsection{Vibrating Device}

When the corona electrode is added, the separation efficiency of the electrostatic cyclone separator is greatly improved. However, after a period of operation, the dust particles will be adsorbed on the wall of the reactor and become thicker and thicker, leading to the decrease of separation efficiency.

Electromagnetic vibration can be used to achieve the purpose of cleaning, as shown in figure 4 . The device mainly consists of three parts: a vibrating rod, a vibrating box and an electromagnet. The main working mechanism: after the electromagnet generates the suction, the vibrating rod is bent by the suction force to the direction of the electromagnet, and the vibration of the outer wall of the electrostatic cyclone is generated.
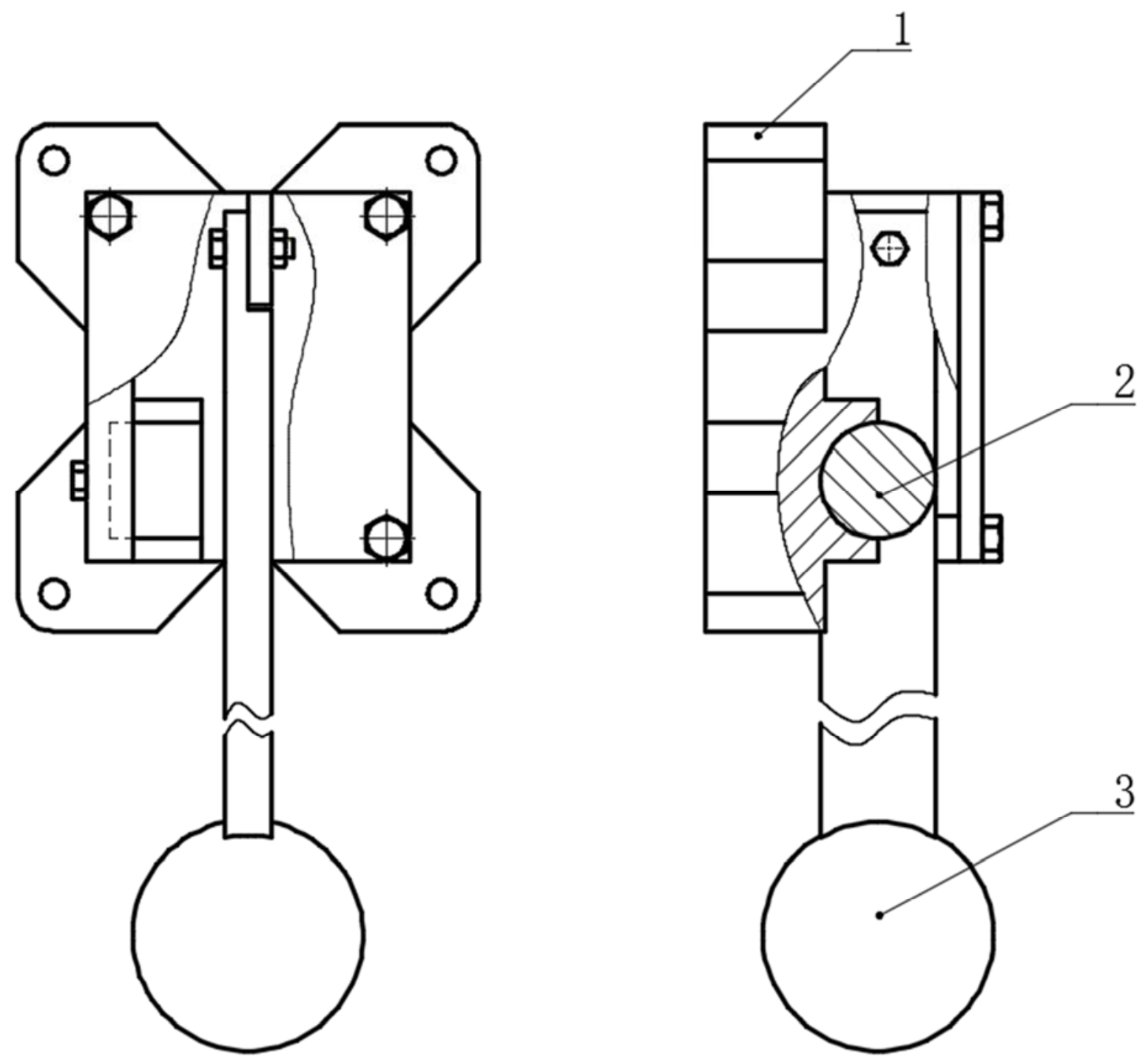

1-vibrating box; 2-electromagnet; 3-vibrating rod

Figure 4. The structure of electromagnetic vibration device.

\subsection{Supporting Device}

After a period of time, the ash bucket must be unloaded to clean ash, so the separator cannot be placed directly on the ground, and supporting structure is necessary. Usually, the ladder is also necessary. The user can conveniently observe the internal condition of the separator from the window or carry out regular maintenance. The device is designed with a support frame and a support plate, which are connected together by bolts.
The supporting frame is composed of three parts: fence, top plate and ladders. The fence is made by steel angle, specifications for $50 \times 50 \times 5$, and the ladders are made by No. 50 steel, at the same time Q235 steel with thickness of $5 \mathrm{~mm}$ was made into top plate. The whole supporting frame is welded, and the structure is shown in Figure 5.

The supporting plate is welded integrally. It plays a role in two aspects: one is to limit the location of the separator, and the other one is the vibration device can be placed on top of it. The structure of the supporting plate is shown in Figure 6. 

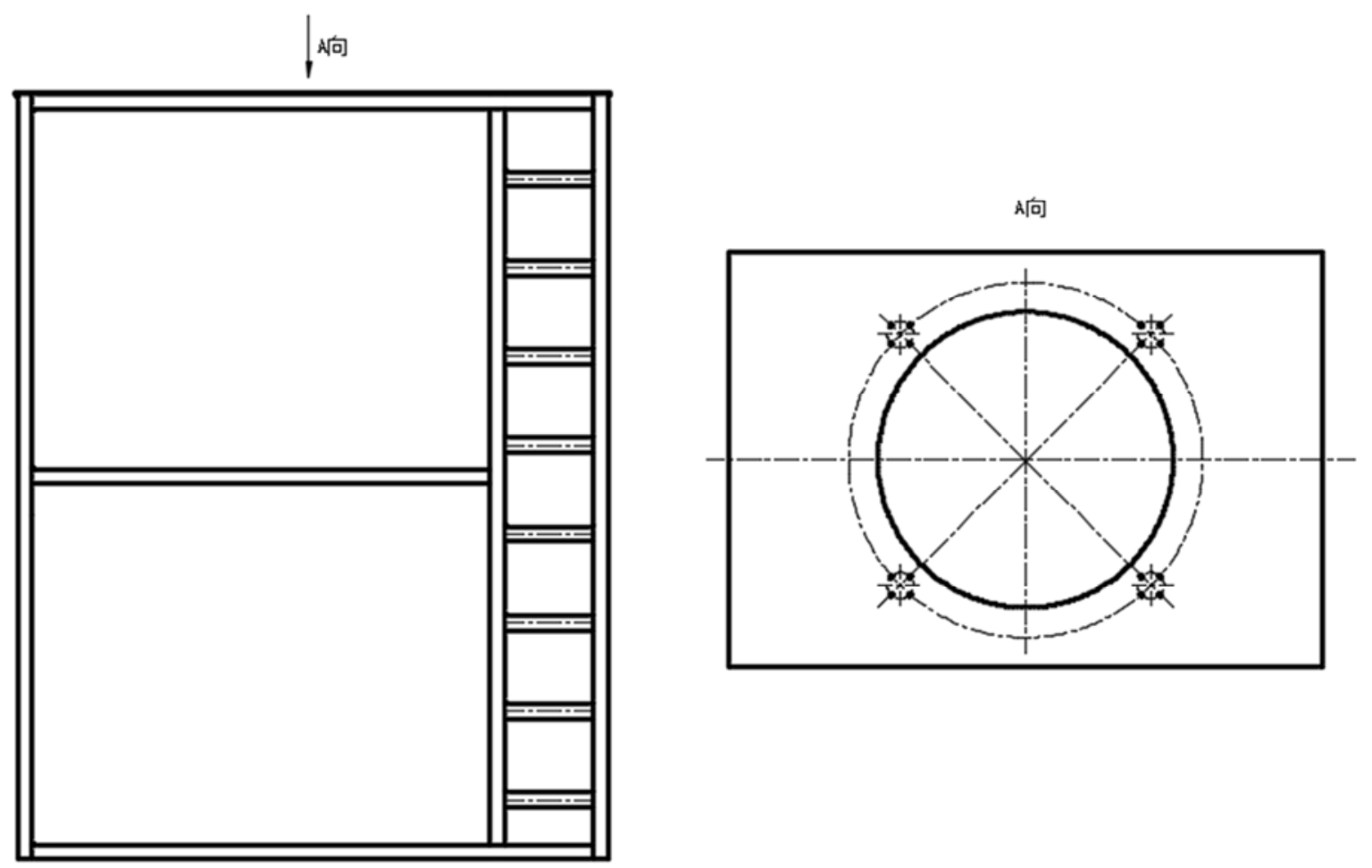

Figure 5. The structure of supporting frame.
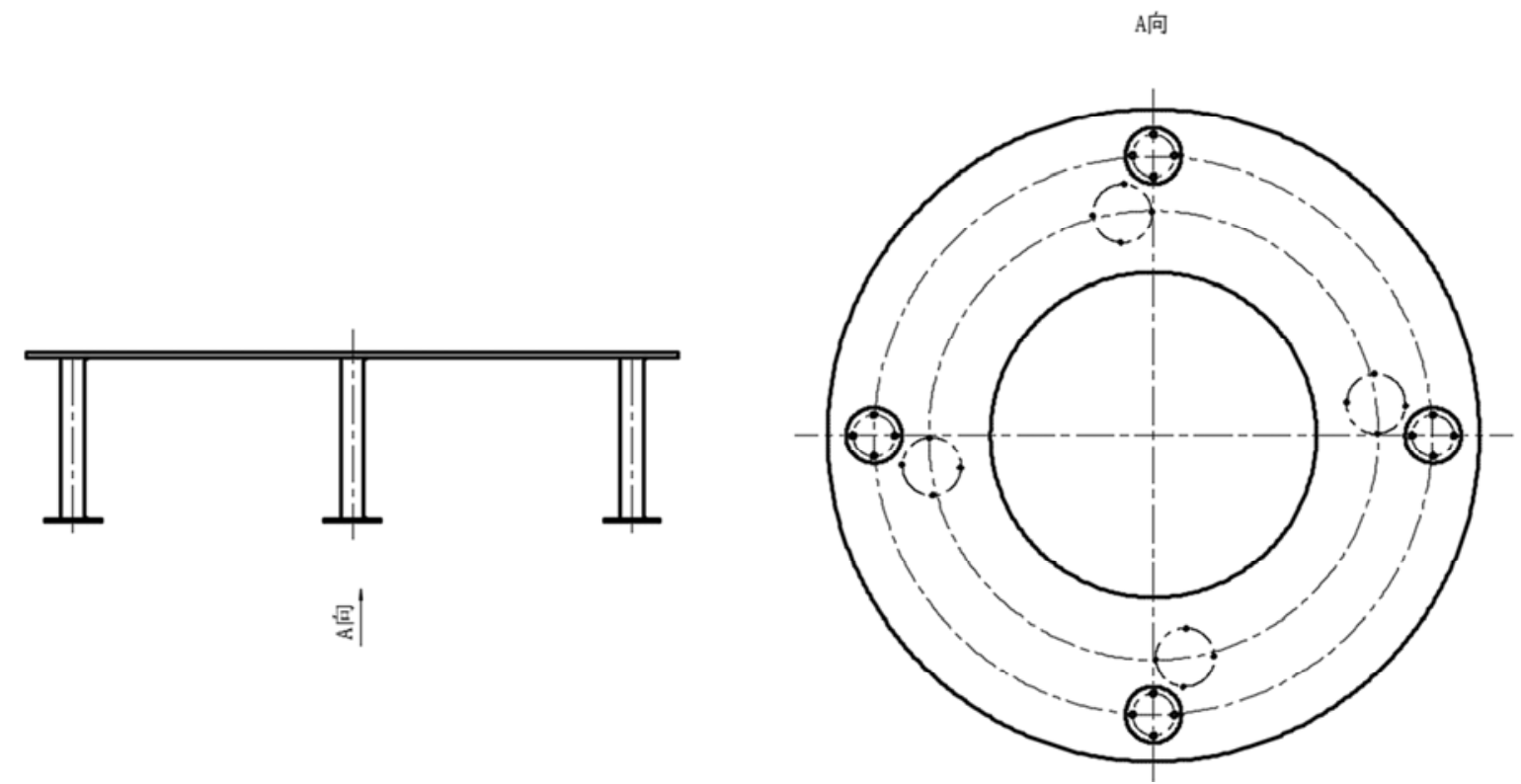

Figure 6. The structure of supporting plate.

\section{Numerical Simulation Analysis}

\subsection{Flow Field Analysis}

Numerical simulation of the flow field in a cyclone separator without a corona electrode is performed using the RNG turbulence model [12]. The analysis results are shown in Figure 7-11.

As shown in Figure 7 and Figure 8, due to the restriction of the wall, the fluid flows into the separator and flows downward. The fluid formed first swirl in the cylinder body. Because the hopper is closed, the lower the air flow must be upward mobility, this creates a second swirl, and have the same rotation direction with the first. Analysis of the whole section, the internal air velocity is approximately symmetrical. Because of the influence of inlet velocity, the separator velocity has a small part of the asymmetry in the periphery of the exhaust pipe. In the periphery of the exhaust pipe velocity is zero. Air flow velocity reaches the maximum value at the entrance, that the turbulence here is obvious. To observe the inner swirl in the separator, it can be 
found that the starting position of the inner vortex is relatively low, which may mean that the particles in the separator will be taken out of the separator. The internal swirl flows upward in a slightly curved path, which may produce a local eddy between the wall and the exhaust pipe, that may affect the pressure drop. It can be seen from the tangential velocity distribution that the tangential velocity near the wall is the largest.

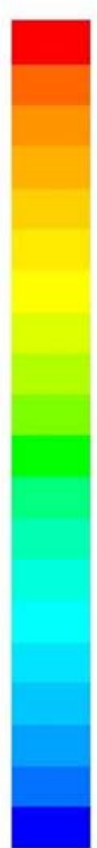
$9.45 e+01$
$8.98 \mathrm{e}+01$
$8.51 e+01$
$8.04 e+01$
$7.57 e+01$
$7.10 e+01$
$6.63 e+01$
$6.16 e+01$
$5.69 e+01$
$5.22 \mathrm{e}+01$
4. $75 \mathrm{e}+01$
4. $28 \mathrm{e}+01$
$3.80 \mathrm{e}+01$
$3.33 e+01$
$2.86 e+01$
$2.39 e+01$
$1.92 e+01$
$1.45 e+01$
$9.81 e+00$
$5.10 e+00$
$3.92 \mathrm{e}-01$
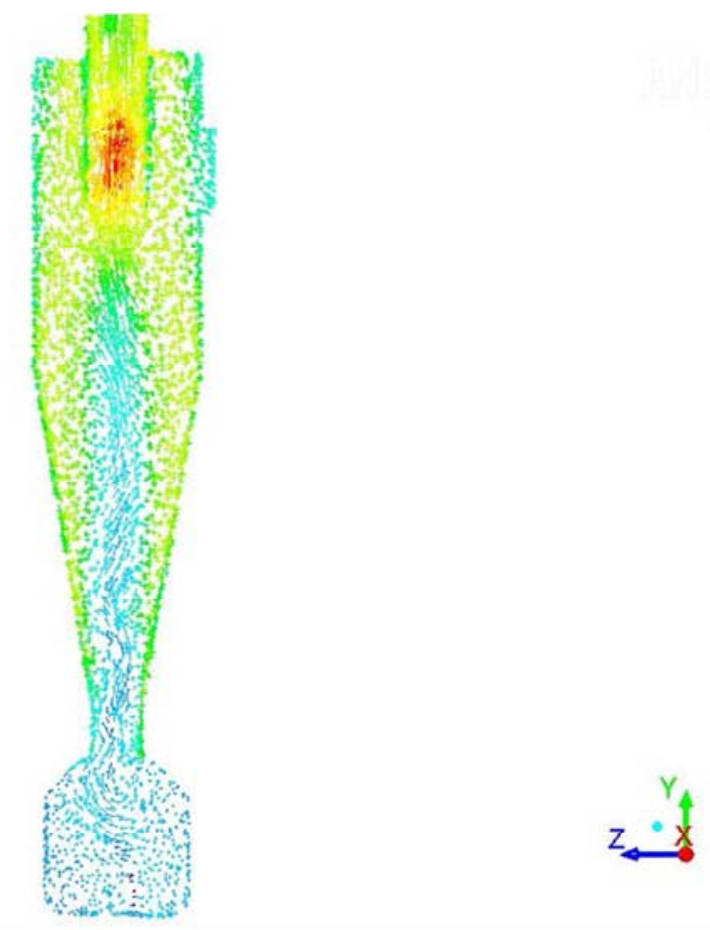

Velocity Vectors Colored By Velocity Magnitude $(\mathrm{m} / \mathrm{s})$

May 19, 2016

ANSYS Fluent 15.0 (3d, pbns, rngke)

Figure 7. The velocity diagram in $X=0$ section.

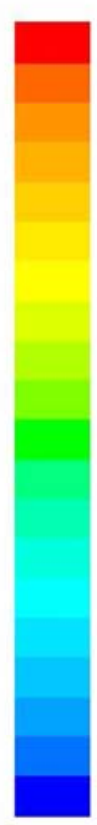

$7.20 \mathrm{e}+01$

$6.48 \mathrm{e}+01$

$5.76 \mathrm{e}+01$

$5.04 \mathrm{e}+01$

$4.32 \mathrm{e}+01$

$3.60 \mathrm{e}+01$

$2.88 \mathrm{e}+01$

$2.16 e+01$

$1.44 \mathrm{e}+01$

$7.23 \mathrm{e}+00$

4. $42 \mathrm{e}-02$

$-7.15 e+00$

$-1.43 e+01$

$-2.15 e+01$

$-2.87 \theta+01$

$-3.59 e+01$

$-4.31 e+01$

$-5.03 e+01$

$-5.75 e+01$

$-6.47 \mathrm{e}+01$

$-7.19 \ominus+01$

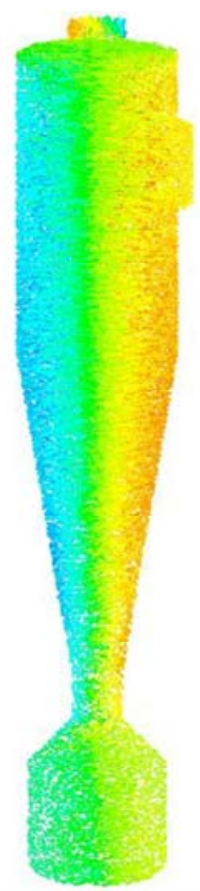

Figure 8. The tangential velocity diagram in $X=0$ section. 


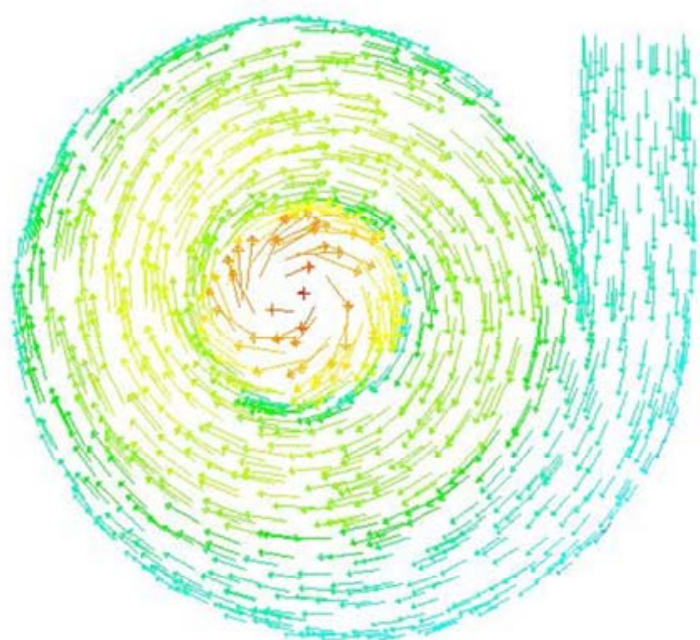

Figure 9. The velocity diagram in inlet middle section.

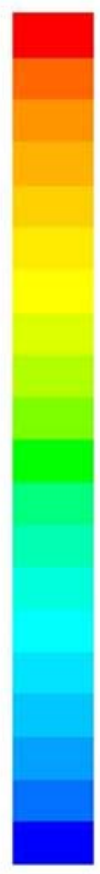
$7.20 \mathrm{e}+01$
$6.48 \mathrm{e}+01$
$5.76 e+01$
$5.04 \mathrm{e}+01$
$4.32 \mathrm{e}+01$
$3.60 \mathrm{e}+01$
$2.88 \mathrm{e}+01$
$2.16 \mathrm{e}+01$
$1.44 \mathrm{e}+01$
$7.23 \mathrm{e}+00$
$4.42 \mathrm{e}-02$
$-7.15 e+00$
$-1.43 e+01$
$-2.15 e+01$
$-2.87 \mathrm{e}+01$
$-3.59 e+01$
$-4.31 e+01$
$-5.03 e+01$
$-5.75 e+01$
$-6.47 e+01$
$-7.19 e+01$

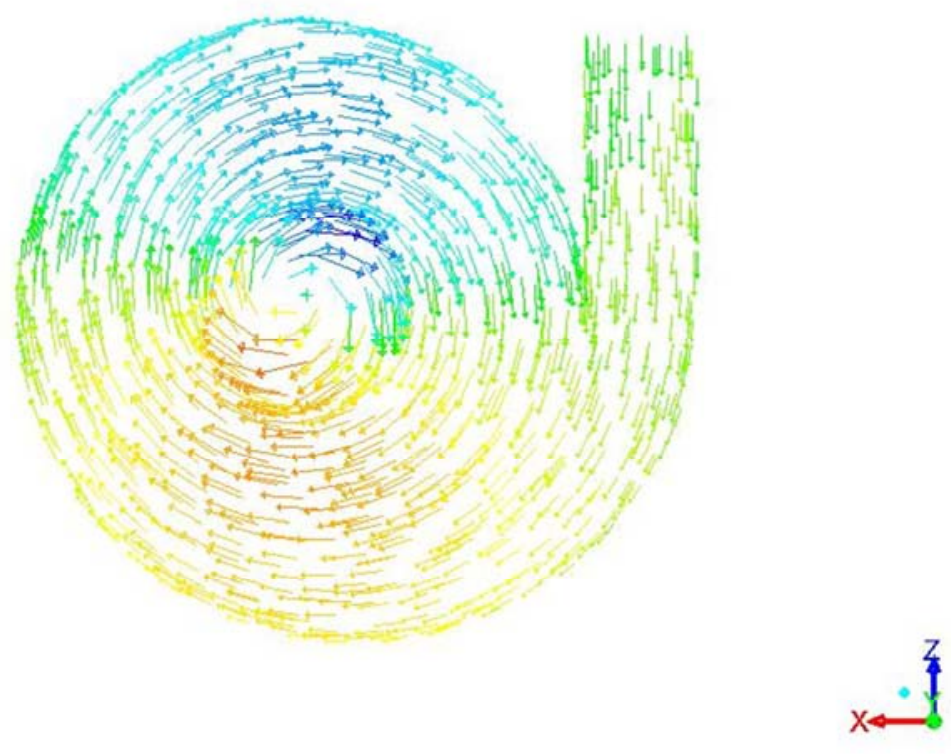



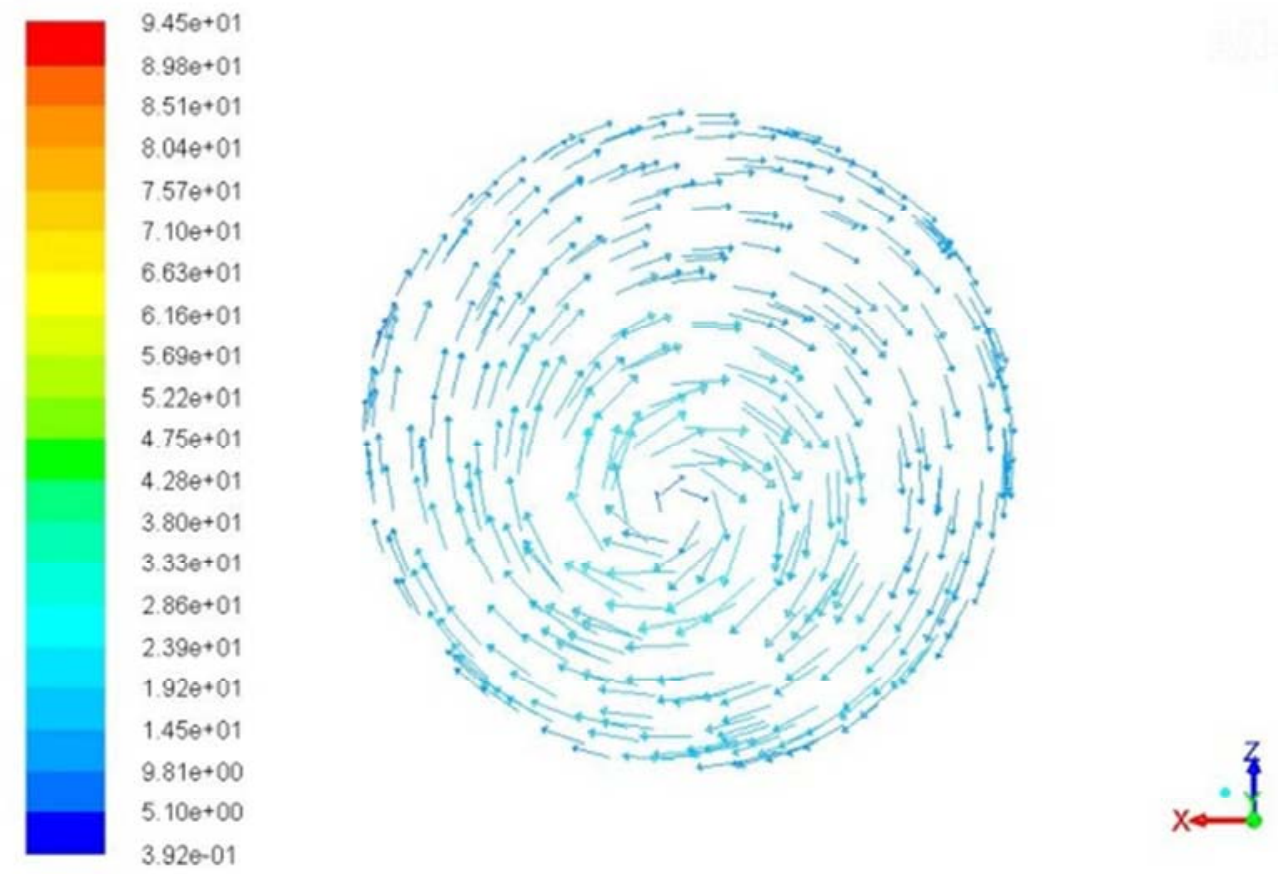

Velocity Vectors Colored By Velocity Magnitude $(\mathrm{m} / \mathrm{s})$

Figure 11. The velocity diagram in Hopper section.

As shown in Figure 9 and Figure 10, the velocity distribution is approximately symmetrical, and the velocity at the wall is small, but the velocity between the wall and the inner vortex is larger than that at the wall. It can be seen from the tangent velocity diagram that the tangential velocity near the wall is greater than the tangential velocity of the inner vortex region, which is the Rankine vortex characteristic, that is, the outer region is stronger, and the inner central area is weaker. The maximum velocity of the middle zone is because the section is just at the inlet of the exhaust pipe, and the turbulence is obvious.

Observe the Figure 11 can know that flow velocity at the hopper section is the smallest, but there are still whirlpools, indicating that the collection of large particles may be entrained out of the separator.

\subsection{Separation Efficiency}

Cutting diameter (When the separation efficiency is $50 \%$, the corresponding dust particle diameter) is used as a standard to evaluate the separation performance of the separator in the simulation analysis. Two kinds of discrete gas and solid are set up to calculate the trajectories of particles in the separator [13, 14]. The inlet velocity is $30 \mathrm{~m} / \mathrm{s}$ and the particle diameter is $0.01 \mathrm{~mm}$. Track the particle, the number of tracking steps set to 20000 , the result is trapped $=959$, incomplete $=1226$. Calculate the efficiency of the current cyclone.

$$
\eta=\frac{\text { trapped }}{\text { number tracked }}=\frac{959}{1226} \approx 78.2 \%
$$

\section{Conclusion}

The electrostatic cyclone separator has the advantages of simple structure, convenient operation and low economic cost. The large particles in the gas can be separated by centrifugal force and after placing the Corona Electrode, and the electric has a good separation effect on the smaller diameter particle, at the same time, the vibration beat facility can clean regularly, it can ensure that the separation efficiency will not fall.

The numerical simulation of Fluent software shows that the velocity distribution in the separator is approximately symmetrical, and there are rankie vortex characteristics. The discrete phase is set up to track the particles in the separator. The results show that the separation efficiency is about $78 \%$. The numerical simulation results are satisfactory, but there will be many problems in the actual operation process, which needs further study.

\section{References}

[1] Zhen Zhao, Guizhen Zhang, Liu Jian. Latest research progresses in catalysts for the purification of exhaust gases from diesel Engines. Chinese Journal of Catalysis, 2008, 29 (3):303-304.

[2] Maimaitimin Kadil, Atagora Tuson. Diesel exhaust hazards and chemical treatments. Journal of Hetian Normal College, 2013, $32(6): 76$.

[3] P Phibin, S Gordon. Experimental fluid dynamic characterization of a cyclone chamber. Experimental Thermal and Fluid Science, 2002, 27 (1):87-96. 
[4] Zhenlan Huang, Zhongmin Zhang, Shixuan Huang, Yuanyuan Gong. Analysis of collections efficiency and energy saving of electrostatic precipitator. Jiangxi Science, 2008, 26 (1):140-143.

[5] Yaxin Su, Bingtao Zhao, Anqiao Zheng. Simulation of turbulent flow in square cyclone separator with different gas exhaust. American Chemical Society, 2011, 50:12162-12169.

[6] R Xiang, SH Park, KW Lee. Effects of cone dimension on cyclone performance. Journal of Aerosol Science, 2001, 32 (4):549-561.

[7] X Li, J Yan, Y Cao, M Ni, K Cen. Numerical simulation of the effects of turbulence intensity and boundary layer on separation efficiency in a cyclone separator. Chemical Engineering Journal, 2003, 95 (1):235-240.

[8] Jihui Chen, Xu Liu. Simulation of a modified cyclone separator with a novel exhaust. Separation and Purification Technology, 2010, 73 (2):100-105.

[9] Alex C. Hoffmann, Louis E. Stein. Gas cyclones and Swirl Tubes: principle, design and operation. Peking, Chemical Industry Publishing House press, 2004.
[10] Xiaobing Zhu, Erpan Wang, Zhiyuan Wu. Design of tail gas purifier for drilling diesel engine. Petroleum and Chemical Equipment, 2016, 9 (16):96-99.

[11] Xinhua Chen. The research about using static electric field to depurate the particulates in the diesel engine exhaust gas. Small Internal Combustion Engine and Motorcycle, 2001, 4 (30):9-10.

[12] Guangji Zhang, Henggen Shen, Hua Li. Flow field analysis of an electrostatic cyclone separator. Engineering for Thermal Energy and Power, 2002, 17 (101):499-501.

[13] Tao Zhou, Chuangang Gu, Tong Wang. Characteristic of separation and numerical simulation of finest particles in cyclone separator. Coal Mine Machinery, 2007, 28 (5):47.

[14] Xinli Wei, Haihong Wang, Dingbiao Wang. Numerical simulation of the paticles' track in a cyclone separator. Journal of Zhengzhou University, 2004, 25 (3):14-17. 